haben. In den Städten, z. B. Kairo, stehen die Dattelpalmen zwischen den Häusern, rings um die Moscheen, in Gärten, oft bis 22 Meter hoch. Die höchste in Kairo stand nahe Kair-Nil, c. 28 Meter hoch; sie wurde 1871 rom Winde umgebrochen und so kam der Patriarch der Dattelpalmen um, don die Gelehrten, welche Napole on I begleiteten, erwähnen, und der etwa 200 Jahre alt war. Strabo giebt an, dass in Aegypten nur im Bezirk Theben gute Datteln wüchsen, wahrscheinlich weil sie nur dort cultivirt wurde.

Gewöhnlich stehen 400 Bäume auf einem Feddan (4500 $\square$ Fuss). Delile giebt in seiner "Flora of Egypt“ an, dass man alte und wenig tragende Bäume verpflanze. Ein Jahr vorher werden zwei Stücke Holz über Kreuz etwa 1 Meter unter dem Wipfel in den Baum getrieben, die Keile und Löcher werden mit Schlamm bedeckt und ein Netz zur Befestigung darum gelegt. Das Ganze wird immer nass gehalten. Am Ende des Winters finden sich Würzelchen unter dem Schlamm, unterhalb desselben wird der Baum dann abgehauen und verpflanzt. (Journal of Applied Science. - American Journal of Pharmacy. 1873. Vol. XLV. 4 th. Ser. Vol. III. pag. 405 s.).

$R$.

\title{
Anwendung des Broms in der Analyse.
}

$\mathrm{Zu}$ der in Band 5. S. 422 gegebenen Notiz von $\mathrm{Vul}$ pius macht Herr Prof. Kämmerer in Nürnberg aufmerksam, dass er schon 1871 in den Berichten der deutschen, chemischen Gesellschaft, Bd. IV S. 218 auf dieselbe Verwendung des Brom's zur Trennung von Nickel und Kobalt aufmerksam gemacht habe. Die Empfehlung von Dr. Vulpius beansprucht auch keineswegs die Neuheit, sondern sollte mehr als Empfehlung der weiteren Verwendung des Brom's dienen. Jedenfalls war jedoch die frühere Angabe von Kämmerer entgangen und geben wir daher sofort die dankenswerthe Berichtigung wieder.

Redaction. 